Trivent Publishing

(C) The Authors, 2016

Available online at http://trivent-publishing.eu/

Engineering and Industry Series

Volume Power Systems, Energy Markets and Renewable Energy Sources in

South-Eastern Europe

\title{
Typical Lightning Protection System for Wind Turbines
}

\author{
Sokratis Pastromas, ${ }^{1}$ Ioannis Naxakis, ${ }^{1}$ Eleftheria Pyrgioti ${ }^{1}$
}

${ }^{1}$ Department of Electrical and Computer Engineering, University of Patras, Greece

\begin{abstract}
Wind turbines are tall structures with heights exceeding 120m. They are usually installed on high altitudes or isolated areas. In these areas, lightning activity could be high and the installation conditions wind turbines are vulnerable to lightning strikes. Furthermore, these areas do not have ideal soil conditions and the efficiency of wind turbine lightning protection system (LPS) can be affected. Additionally, the LPS shall be compatible with IEC61400-24 as minimum; furthermore, the earthing system shall keep step and touch voltages within the limits defined in IEEE80-2000. Considering the high amounts of wind turbines installed annually and also the limited project time-schedules, it is needed to have a typical LPS for wind turbines that complies with the relevant standards; based on this, wind turbines are armoured against lightning strikes. In case of non-effective LPS, damages can be caused in vital wind turbine components like blades which can lead to downtime and loss of energy production. This paper will present a typical LPS for wind turbines. This system can be applied in every site condition up to a pre-defined level, provided that it respects local legislation and can protect the structure in a reliable way. This proposal does not replace the site specific design of LPS but can be used as basis for further adaption in local conditions.
\end{abstract}

This is an Open Access article distributed in accordance with the Creative Commons Attribution Non Commercial (CC-BY-NC-ND 4.0) license, which permits others to copy or share the article, provided original work is properly cited and that this is not done for commercial purposes. Users may not remix, transform, or build upon the material and may not distribute the modified material (http://creativecommons.org/licenses/by-nc/4.0/) 


\section{Keywords}

ATP-EMTP; earthing system; LPS; wind turbine

\section{Introduction}

In recent years, there has been continuous development of wind turbines. Wind turbines are increasing in installed power, size and thus in rotor diameter. This development (apart from the positive effect it has on the industry because of low energy costs) causes these structures to be vulnerable in lightning strikes. This is caused by their installation areas, usually in high mountains or in isolated areas where lightning activity is usually medium to high. Such areas are also of poor soil conditions, usually rocky areas causing extra needs for earthing infrastructure of the wind turbines and of the whole wind farm earthing system. The business activity of wind energy sector is annually getting higher; this is an effect mainly driven by $2020 \mathrm{EU}$ targets and also by the global commitment of developed countries to reduce $\mathrm{CO}^{2}$ emissions. The combination of high business activity and non-ideal weather conditions for soil investigation leads to the need of a typical Lightning Protection System of wind turbines (LPS). This paper presents an earthing system which can be considered the basis for a wind turbine earthing system and complies with the IEEE Std.80-2000. The implementation of this system can ensure the equipment reliable protection and also the safety of the personnel. The earthing system presented hereto is part of a wind turbine LPS and is a generic one, not wind turbine related. It will be proven that this system is compliant with IEEE St.80-2000 and IEC62305-1 standards while with minor add-ons it can be extended to wind farm areas.

\section{Modelling of WTG typical earthing system}

In recent years, there has been a continuous development of wind turbines. Wind turbines are increasing in installed power, size and thus in rotor diameter.

As wind turbines increase their rated power, they become more vulnerable to lightning strikes since they are increasing in height. Wind turbines are usually placed on high altitudes and isolated locations as well as on flat countryside areas. In these areas, there is usually a medium to high level of lightning activity which directly affects wind turbines. These areas are usually of low quality soil conditions with resistivity exceeding $500 \Omega \mathrm{m}$. The earthing system part of wind turbine LPS that is presented here applies for soil resistivity up to a specific soil resistivity level that will be defined in the next section. 
The earthing system that is studied here is Type B and consists of horizontal copper electrodes with diameter of $17 \mathrm{~mm}$ and vertical ones of $20 \mathrm{~mm}$ diameter. The earthing system is installed at a depth of $2 \mathrm{~m}$. The foundation was considered to have resistivity of $\rho \mathrm{fnd}=50 \Omega \mathrm{m}$. The surrounding soil resistivity of the ground was considered to be $\rho \mathrm{s} 1=500 \Omega \mathrm{m}$ and $\rho \mathrm{s} 1=1000 \Omega \mathrm{m}$ while the earthing system will be installed inside the wind turbine foundation as shown in (Fig.1). The influence of the earthing interconnection wire positively affects the performance of the wind turbine that has been hit by lightning strike.

The wind turbine earthing system was simulated in ATP-EMTP by using the circuit-theory due to the simplicity, the capability to describe the soil ionization; furthermore, it was simulated because this theory can take into account the interference between the earthing electrodes. Because the aim of the present work is to propose a typical earthing of a wind turbine LPS, the effect of the tower which is steel for the large majority of the wind turbines will be considered.

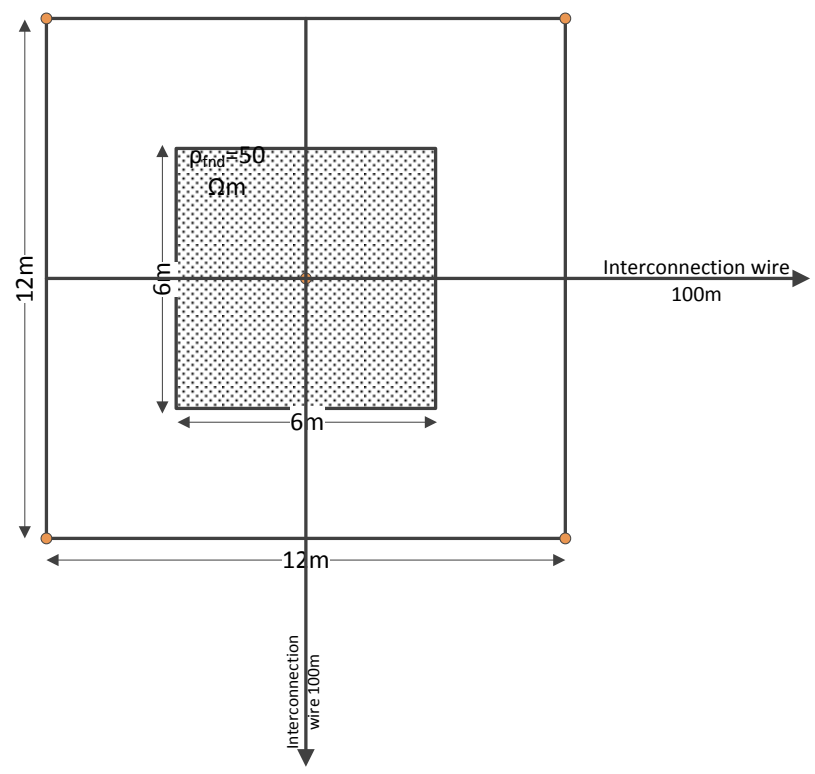

Fig. 1. Wind turbine earthing system

The formulas that have been considered are the following ones for the horizontal earthing electrodes:

$L i \approx\left(\frac{l_{i} \mu_{0}}{2 \pi}\right)\left(\ln \left(\frac{(2 l i)}{\alpha}\right)-1\right)$ 


$$
\begin{aligned}
& r_{i}=\frac{\rho}{\left(2 \pi l_{i}\right)}\left[\frac{2 h+a}{l_{i}}+\ln \frac{l_{i}+\sqrt{\left(l_{i}^{2}+a^{2}\right)}}{a}-\sqrt{1+\left(\frac{a}{l_{i}}\right)^{2}}+\ln \frac{l_{i}+\sqrt{\left(l_{i}^{2}+4 h^{2}\right)}}{2 h}-\sqrt{1+\left(\frac{2 h}{l_{i}}\right)^{2}}\right] \\
& C_{i}=\frac{2 \pi \varepsilon l_{i}}{\frac{a_{i}}{l_{i}}+\ln \frac{l_{i+\sqrt{l_{i}^{2}+a_{i}^{2}}}^{a_{i}}}{a_{i}} \sqrt{1+\left(\frac{a_{i}}{l_{i}}\right)^{2}}} \\
& G_{i}=\frac{C_{i}}{\varepsilon \rho} \\
& \alpha_{i}=\frac{\rho \Delta I_{i}}{2 \pi E_{c} l_{i}}
\end{aligned}
$$

For the vertical electrodes following equations are used:

$G^{-1}=\frac{\rho}{2 \pi l} \log \frac{2 l_{l}}{a_{i}}$

$C=2 \pi \varepsilon \log \frac{2 l}{a_{i}}$

$L=\frac{\mu_{0}}{2 \pi} \log \frac{2 l}{a_{i}}$

li= length of $i$ section of earthing electrode

$\mu 0=$ permeability of air (vacuum)

$\varepsilon=$ dielectrilic permeability of the soil

$\rho=$ soil resistivity

$\mathrm{h}=$ depth of electrode installation

ai=equivalent electrode radius considering ionization phenomenon

The earthing system has been simulated in ATP-EMTP and is shown in (Fig.2). 


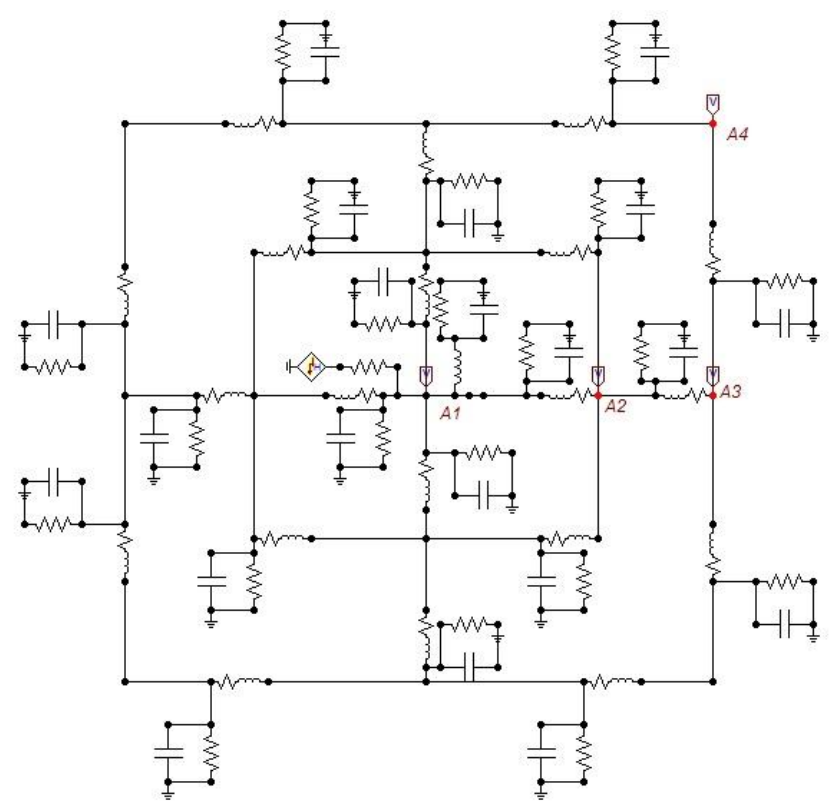

Fig. 2. Wind turbine earthing system in ATP-EMTP

With the use of the previous formulas (1)-(8) and considering the soil ionization, the model parameters in ATP-EMTP are calculated and the results are summarized in Table 1.

Table 1. Model parameters for the WTG

\begin{tabular}{|l|l|l|l|l|}
\hline & \multicolumn{4}{|c|}{$\begin{array}{c}\text { WTG model parameters } \\
\text { (with soil ionization) }\end{array}$} \\
\hline & $\mathrm{L}(\mu \mathrm{H})$ & $\mathrm{R}(\Omega)$ & $\mathrm{C}(\mathrm{nF})$ & $\mathrm{G}-1(\Omega)$ \\
\hline $\mathrm{l}=3 \mathrm{~m}$ & 3.33 & 15.68 & 0.92 & 2.39 \\
\hline $\mathrm{l}=6 \mathrm{~m}$ & 7,50 & 9,16 & 0,83 & 2.65 \\
\hline $\mathrm{l}=2 \mathrm{~m}$ (ver.) & 1.04 & - & 0.17 & 1.20 \\
\hline
\end{tabular}

\section{Simulation results and extrapolation}

The simulation model of a wind turbine typical earthing system is shown in (Fig. 2). The simulation of the lightning current that hits directly a wind turbine will be implemented in the software by using Heidler current source of I $=150 \mathrm{kA}$ and two types of waveforms $1.2 / 50 \mu$ s and $19 / 485 \mu \mathrm{s}$. The simulation will be executed for two different types of surrounding soil resistivity of $\rho s 1=500 \Omega \mathrm{m}$ and $\rho s 2=1000 \Omega \mathrm{m}$. 
A. Case 1: Ip=150kA $-1.2 / 50 \mu \mathrm{s}, \rho_{s 1}=500 \Omega \mathrm{m}$

The first simulation was executed considering as lightning current $\mathrm{Ip}=150 \mathrm{kA}$ and time parameters $1,2 / 50 \mu$ s simulating a lightning strike and soil resistivity of $500 \Omega \mathrm{m}$. As shown in (Fig.3) and (Fig.4), the reported voltage levels at nodes $\mathrm{A} 1-\mathrm{A} 4$ in the affected wind turbine are:

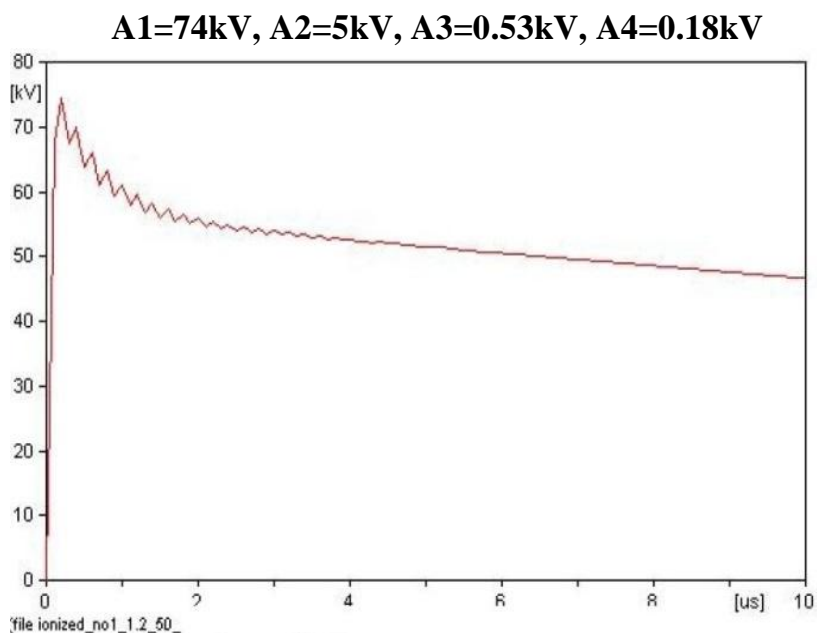

Fig. 3. Voltage at earthing grid node A1

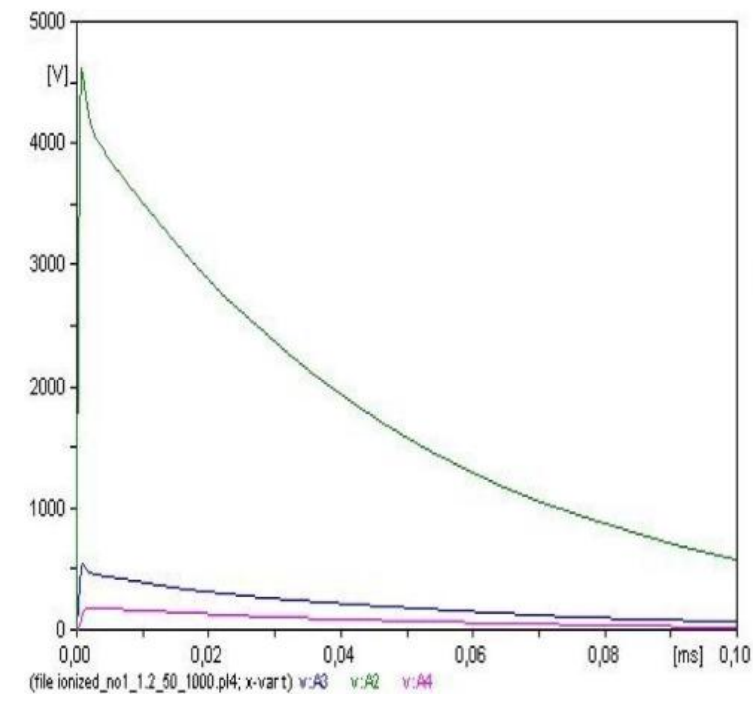

Fig. 4. Voltage at earthing grid nodes A2, A3, A4 


\section{B. Case 2: $I p=150 k A-1,2 / 50 \mu s, \rho_{s 2}=1000 \Omega m$}

In this case, the simulation was executed considering as lightning current $\mathrm{Ip}=150 \mathrm{kA}$ and time parameters $1.2 / 50 \mu \mathrm{s}$ simulating a lightning strike and soil resistivity of $1000 \Omega \mathrm{m}$. The results remain the same for nodes A1 and A2 while for nodes A3 and A4, they are depicted in (Fig. 5). For the specific case, the reported voltage levels at nodes A1-A4 are:

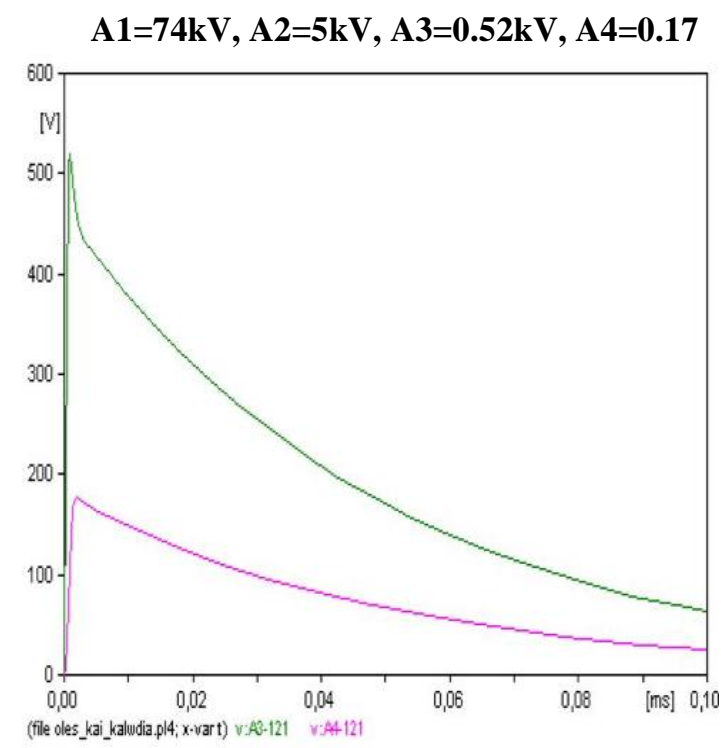

Fig. 5. Voltage at earthing grid nodes A3, A4

\section{Case 3: $I p=150 \mathrm{kA}-19 / 485 \mu \mathrm{s}, \rho_{s I}=500 \Omega \mathrm{m}$}

In this case, the simulation was executed considering as lightning current $\mathrm{Ip}=150 \mathrm{kA}$ and time parameters $19 / 485 \mu \mathrm{s}$ and soil resistivity of $500 \Omega \mathrm{m}$. The results are different for node A1 (Fig. 6), while for nodes A2, A3 and A4, they are depicted in (Fig. 7). For the specific case, the reported voltage levels at nodes A1-A4 are:

$$
\mathrm{A} 1=55 \mathrm{kV}, \mathrm{A} 2=4 \mathrm{kV}, \mathrm{A3}=0.40 \mathrm{kV}, \mathrm{A} 4=0.17 \mathrm{kV}
$$




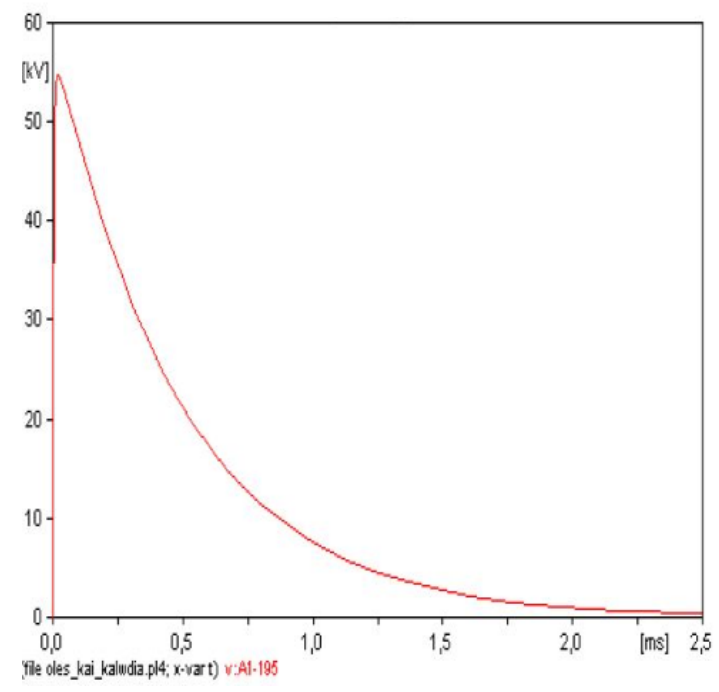

Fig. 6. Voltage at earthing grid nodes A1

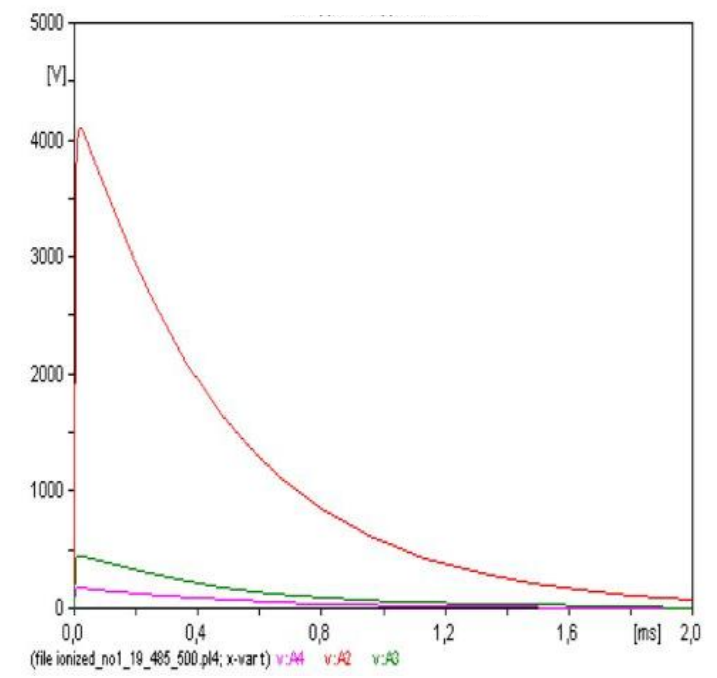

Fig. 7. Voltage at earthing grid nodes A2, A3, A4

D. Case 4: $I p=150 k A-19 / 485 \mu s, \rho_{s 2}=1000 \Omega m$

In this case, the simulation was executed considering as lightning current $\mathrm{Ip}=150 \mathrm{kA}$ and time parameters $19 / 485 \mu$ s and soil resistivity of $1000 \Omega \mathrm{m}$. The results remain the same with case 3 for nodes A1 and A2 (Fig. 8 and Fig. 9) while for nodes A3 and A4 they are depicted in (Fig.10). For the specific case, the reported voltage levels at nodes A1-A4 are: 
$\mathrm{A} 1=55 \mathrm{kV}, \mathrm{A} 2=4 \mathrm{kV}, \mathrm{A} 3=0.43 \mathrm{kV}, \mathrm{A4}=0.17 \mathrm{kV}$

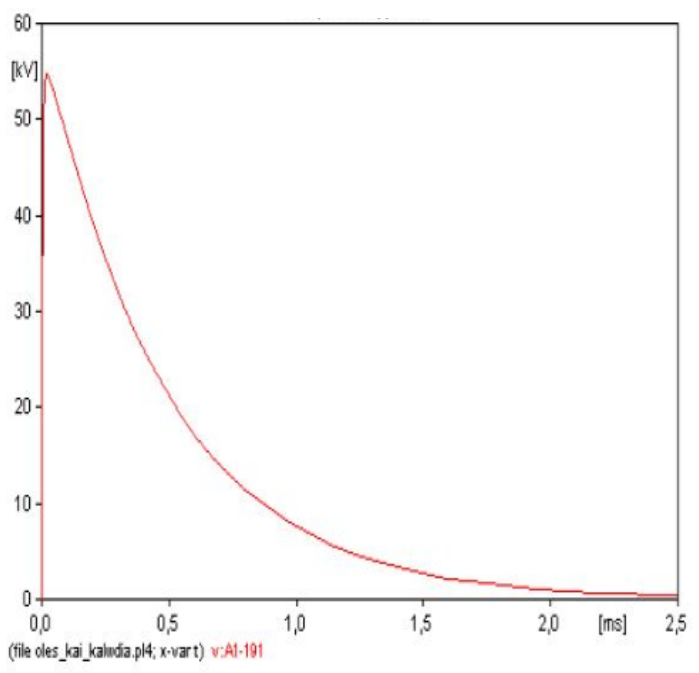

Fig. 8. Voltage at earthing grid nodes A1

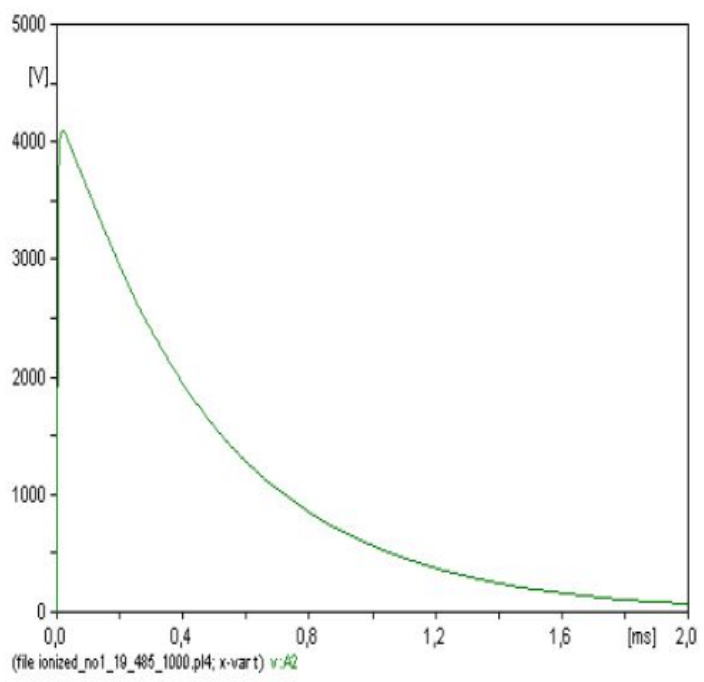

Fig. 9. Voltage at earthing grid nodes A2 


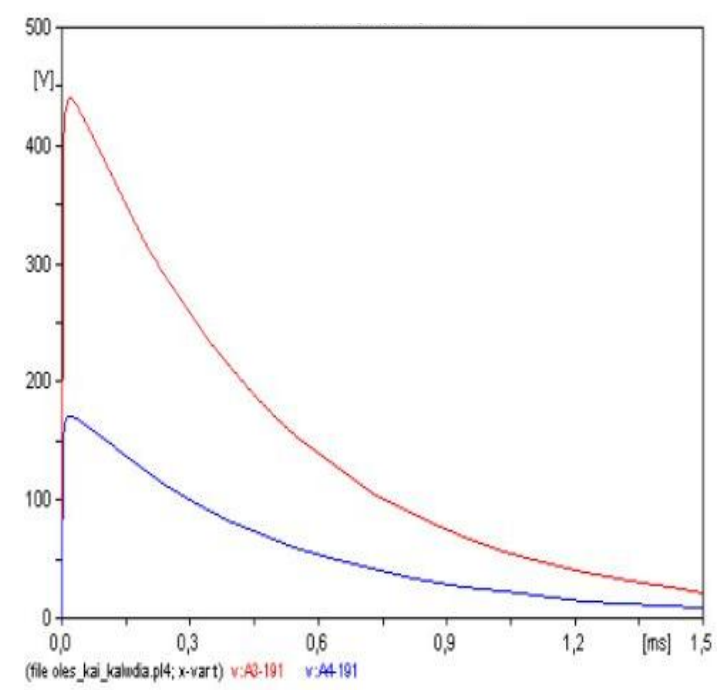

Fig. 10. Voltage at earthing grid nodes A3 and A4

\section{Assessing the reliability of the LPS}

In the previous section, the performance of the typical earthing system of a wind turbine LPS was simulated with the use of ATP-EMTP. It was noticed that at the point of attack it faced high voltages which can be approximately $54 \mathrm{kV}$ which are significantly lowering some meters away. In order for the reliability of the earthing system to be assessed, its effectiveness to protect the personnel against step and touch voltages need to be verified. Step and touch volutages could be phased during an electrical earth fault following the requirements of IEEE. For this specific case, the following formulas exist:

$$
\begin{aligned}
& E_{\text {step }, 70}=\left(1000+6 C_{S} \rho_{s}\right) 0,157 / \sqrt{t_{s}} \\
& E_{\text {touc } h, 70}=\left(1000+1,5 C_{s} \rho_{s}\right) 0,157 / \sqrt{t_{s}} \\
& E m=(\rho \operatorname{IgKiKm}) / L m \\
& E s=(\rho \operatorname{IgKiKs}) / L s \\
& G P R=\operatorname{IgRg}
\end{aligned}
$$

$\mathrm{Cs}=$ surface layer derating factor $\rho s=$ surface material resistivity in $\Omega \mathrm{m}$ 
ts=fault clearance time

$\rho=$ soil resistivity

Ig= fault current

$\mathrm{Ki}=$ irregularity factor

$\mathrm{Km}=$ geometric space factor

$\mathrm{Lm}=$ effective buried length of grounding system conductor

$\mathrm{Ks}=$ geometrical factor

Ls=effective buried conductor legth

$E m=m e s h$ voltage, the maximum touch voltage within a mesh of a ground grid

Estep, $70=$ step voltage

Etouch, $70=$ touch voltage

For the specific earthing design considering fault clearance time less than $0.5 \mathrm{sec}$, fault current of $1000 \mathrm{~A}, \rho s 1=500 \Omega \mathrm{m}$ without surface material and by using the formulas (9)-(12) below, results appear for the maximum permitted limits for step and touch voltages:

$E_{\text {step }, 70}=888.13 \mathrm{~V}$

$E_{\text {touc } h, 70}=388.56 \mathrm{~V}$

In order an earthing grid to be safe it is needed below conditions to be fulfilled:

$E m<E_{\text {touc } h}$

Es $<E_{\text {step }}$

$G P R<E_{\text {touc } h}$

$G P R<E_{\text {step }}$

For the specific case the earthing system is not safe since the calculated values are as below:

$E m=3927.88 \mathrm{~V}$ 
$E s=3106.24 \mathrm{~V}$

$G P R=23948.32 \mathrm{~V}$

In order for the system to be considered safe, the fault conditions and the earthing conditions need improvement. For that purpose, the improvement of soil with surface material of $\rho \mathrm{s}=3000 \Omega \mathrm{m}$ with thickness $20 \mathrm{~cm}$, fault clearance time of $\mathrm{tf}=0.08 \mathrm{sec}$ and fault current $\mathrm{If}=0.050 \mathrm{kA}$ was considered. Now, the maximum permitted limits are:

$E_{\text {step }, 70, \rho s 1}=9017.20 \mathrm{~V}$

$E_{\text {touc } h, 70, \rho s 1}=2670.61 \mathrm{~V}$

After the improvement in the soil conditions and changing the fault parameters the new results are:

$E m_{\rho s 1}^{\prime}=294.93 \mathrm{~V}$

$E s_{\rho s 1}^{\prime}=204.68 \mathrm{~V}$

$G P R_{\rho s 1}^{\prime}=1371,43 \mathrm{~V}$

Now all the conditions are fulfilled and the earthing system is safe. The same conditions will be evaluated considering soil conditions of $\rho s 2=1000 \Omega \mathrm{m}$. The maximum permitted limits are:

$E_{\text {step }, 70, \rho s 2}=9323.06 \mathrm{~V}$

$E_{\text {touc } h, 70, \rho s 2}=2747.07 \mathrm{~V}$

After the improvement in the soil conditions and changing the fault parameters the new results are:

$E m_{\rho s 2}^{\prime}=589.87 \mathrm{~V}$

$E s_{\rho s 2}^{\prime}=409.36 \mathrm{~V}$

$G P R_{\rho s 2}^{\prime}=2742.86 \mathrm{~V}$ 
Thus the modified earthing system design and electrical fault parameters fulfil the requirements and is safe for the personnel (protecting against step and touch voltages).

\section{Conclusion}

This paper presented a typical earthing system part of a wind turbine LPS with hub height of $70 \mathrm{~m}$ and tower base of $7.5 \mathrm{~m}$ diameter. This earthing system can be considered the basis in order to be implemented for soil conditions up to $1000 \Omega \mathrm{m}$ since its reliable operation both for the equipment and personnel can be secured. This system is designed with specific fault parameters regarding the permitted fault current and clearance time while it was proved via simulation results that it dissipates effectively the lightning current that is led by the wind turbine internal LPS. This earthing system can be adapted in more severe soil conditions by extending the surface and density of the earthing grid in order to achieve site specific or local legislation compliance. In case the fault parameters are different, a new design is required, as well as the re-assessment of the earthing system effectiveness, while it needs to be improved accordingly.

\section{References}

IEC 61400-24, "Wind turbines Part 24: Lightning protection", edition 1.0, 2010. Nikolakopoulos Ch., "Transient behaviour of earthing", Diploma Thesis, University of Patras, Patras, February 2013.

Pastromas S., Nikolakopoulos Ch., Peppas G., Naxakis I., Pyrgioti E., "Effect on GPR of earthing connection between wind turbines" (paper presented in 2nd International Conference on Renewable Energy for Developing Countries, REDEC 2014, Lebanon, November 26-27, 2014).

ANSI/IEEE Std 80-2000, "IEEE Guide for Safety in AC substation grounding", 2000.

Pastromas S., Pyrgioti E., "Two types of earthing of lightning protection for wind turbines" (paper presented in 29th International Conference on Lightning Protection", ICLP2008, Uppsala, Sweden, June 2008). IEC 62305-1, "Protection against lightning - Part 1: General principles", 2006. 